Rev Esp Casos Clin Med Intern (RECCMI). 2020 (Ago); 5(2): 87-89

\title{
Síndrome de Marfan de presentación atípica, reflejo de una nueva mutación
}

\author{
Cristina Sardiña-González', Laura Villaverde-Piñeiro², Raúl Franco-Gutiérrez ${ }^{3}$, Marcos Nicolás-Cicerchia ${ }^{4}$, José López-Castro' \\ ${ }^{1}$ Servicio de Medicina Interna. Hospital Público de Monforte. Monforte de Lemos (Lugo). España \\ ${ }^{2}$ Servicio de Farmacia. Hospital Público de Monforte. Monforte de Lemos (Lugo). España \\ ${ }^{3}$ Servicio de Cardiología. Hospital Universitario Lucus Augusti. Lugo. España \\ ${ }^{4}$ Servicio de Cardiología. HeathInCode. A Coruña. España
}

Recibido: 11/05/2020

Aceptado: 28/07/2020

En línea: 31/08/2020

Citar como: Sardiña-González C, Villaverde-Piñeiro L, Franco-Gutiérrez R, Nicolás-Cicerchia M, López-Castro J. Síndrome de Marfan de presentación atípica, reflejo de una nueva mutación. Rev Esp Casos Clin Med Intern (RECCMI). 2020 (Ago); 5(2): 87-89. doi: 10.32818/reccmi.a5n2a11.

Cite this as: Sardiña-González C, Villaverde-Piñeiro L, Franco-Gutiérrez R, Nicolás-Cicerchia M, López-Castro J. Marfan syndrome: atypical presentation, reflection of a new FBN1 mutation. Rev Esp Casos Clin Med Intern (RECCMI). 2020 (Ago); 5(2): 87-89. doi: 10.32818/reccmi.a5n2a11.

Autor para correspondencia: José López-Castro. jlcastro126@hotmail.com

\section{Palabras clave \\ $\triangleright$ Síndrome de Marfan \\ $\triangleright$ Nueva mutación \\ $\triangleright$ Edema pulmonar agudo}

\begin{tabular}{l} 
Keywords \\
\hline Marfan syndrome \\
$\triangleright$ New mutation \\
$\triangleright$ Acutelung edema \\
\hline
\end{tabular}

\section{Resumen}

El síndrome de Marfan (SM) es un desorden del tejido conectivo que afecta a los sistemas musculoesquelético, cardiovascular y ocular. Presentamos el caso de una paciente diagnosticada de SM tras sufrir un cuadro de edema pulmonar agudo, objetivándose a posteriori una nueva mutación del gen FBN1.

Abstract
Marfan syndrome (MS) is an inherited connective tissue disorder that affects the skeletal, cardiovascular, and
muscular systems of the eye. We present a MS case with an atypical presentation as acute lung edema that
reflects a new FBN1 mutation.

\section{Puntos destacados}

$\triangleright$ El síndrome de Marfan está infradiagnosticado. Suele reconocerse por sus características fenotípicas.

$\triangleright$ Se han detectado nuevas mutaciones en el gen FBN1 que podrían sugerir diferentes tipos de presentación clínica o, incluso, un patrón evolutivo distinto del síndrome.

\section{Introducción}

El síndrome de Marfan (SM) es un trastorno hereditario autosómico dominante del tejido conectivo que afecta a los sistemas musculoesquelético, cardiovascular y ocular. Tiene una incidencia de 1/10.000 nacidos vivos ${ }^{1,2,}$ sin influencia de etnia ni sexo. La causa genética más frecuente (> 90\%) subyace en una mutación heterocigota del gen FBN1, localizado en el cromosoma 15, que codifica para la fibrilina-1. En otros casos las mutaciones pueden estar presente en los genes TGFBR2 y TGFBR1'. Aunque la mayoría de los individuos con síndrome de Marfan tiene antecedentes familiares, aproximadamente el 25\% lo adquiere como resultado de una mutación de novo ${ }^{1,2}$. El diagnóstico se suele basar en criterios clínicos, pero la forma de presentación fenotípica es muy diversa. Es importante identificar los casos de riesgo, teniendo en cuenta la disponibilidad de tratamientos médicos y quirúrgicos que mejoran significativamente la esperanza de vida. Los estudios moleculares pueden proporcionar un diagnóstico etiológico en los pacientes con formas de presentación clínica atípicas o más leves, y contribuyen al manejo preventivo de portadores y el consejo genético.

En este artículo, describimos un caso con una forma de presentación clínica atípica, en el que se ha identificado una variante en la mutación del gen FBN1.

\section{Caso clínico}

\section{Antecedentes}

Mujer de 34 años, natural de Brasil y residente en España en los últimos 13 años. Consumía tabaco y otras drogas por vía inhalada, sin comorbilidades conocidas. En el año 2015 padeció tuberculosis pulmonar, correctamente tratada y curada. Como antecedentes familiares destacaba un hermano fallecido a los 25 años en su país de origen con el diagnóstico de estenosis mitral severa y miocardiopatía dilatada, pero sin otros antecedentes familiares de patología cardíaca, aórtica, ocular o musculoesquelética. 
Sardiña-González C, Villaverde-Piñeiro L, Franco-Gutiérrez R, Nicolás-Cicerchia M, López-Castro J.

Síndrome de Marfan de presentación atípica, reflejo de una nueva mutación

\section{Exploración física}

Acudía a Urgencias por un cuadro de 96 horas de evolución consistente en disnea, taquipnea y tos productiva con expectoración amarilla. Negaba dolor torácico y/o fiebre. Presentaba tensión arterial de 133/85 mmHg, frecuencia cardíaca de 110 lpm, saturación de oxígeno del 87\% respirando aire ambiente y frecuencia respiratoria de 24 rpm, y sin fiebre. En el examen físico se apreciaba cráneo largo y estrecho, enoftalmos e hipoplasia malar leve. Aracnodactilia (Figura 1), pectus excavatum y estrías cutáneas en mamas (Figura 2), abdomen y espalda. En la auscultación cardiopulmonar presentaba soplo sistólico mitral y crepitantes bibasales, sin otras alteraciones relevantes.

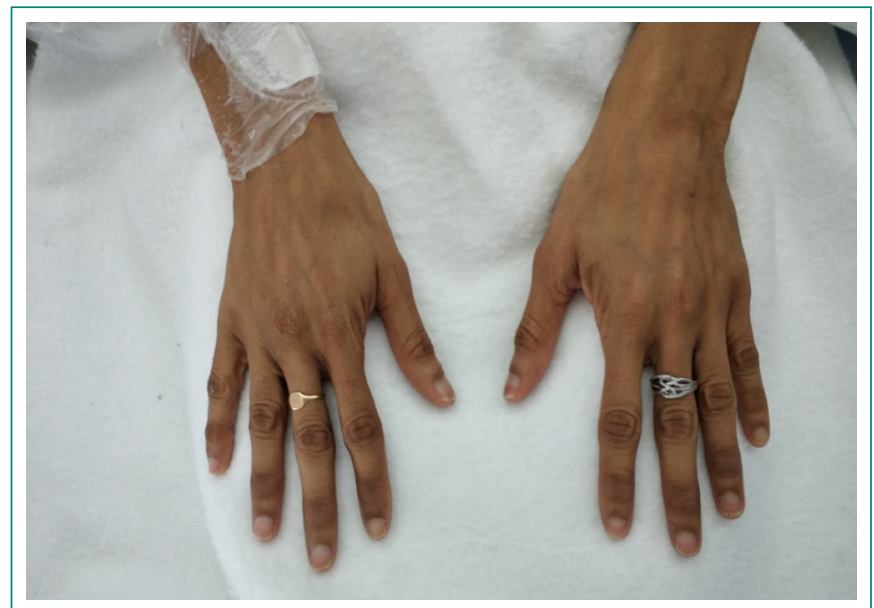

Figura 1. Aracnodactilia

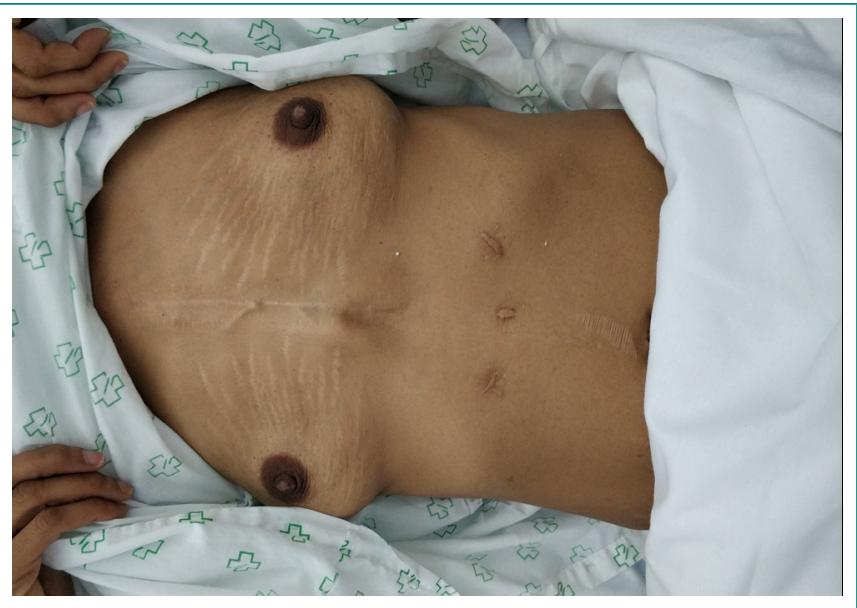

Figura 2. Pectus excavatum y estrías mamarias

\section{Pruebas complementarias}

En las pruebas de laboratorio destacaban leucocitosis con desviación izquierda y elevación de reactantes de fase aguda, y datos de insuficiencia respiratoria global en la gasometría arterial basal. En la radiografía de tórax realizada en Urgencias se objetivaban infiltrados intersticiales bilaterales y ausencia de cardiomegalia. En el electrocardiograma presentaba taquicardia sinusal, a 110 lpm, con datos indirectos de crecimiento de cavidades izquierdas.

\section{Evolución}

Ante estos hallazgos, se interpretó como probable una neumonía comunitaria bilateral complicada con edema pulmonar agudo e insuficiencia respiratoria aguda secundaria, que precisó ingreso en la unidad de cuidados intensivos.
Recibió tratamiento antibiótico intravenoso y deplectivo además de ventilación mecánica no invasiva. La mejoría fue progresiva.

Ya en situación de estabilidad clínica, se realizaron ecocardiografías transtorácica (ETT) y transesofágica (ETE) en las que se objetivó una válvula mitral con gran redundancia de velos (tipo Barlow), con insuficiencia mitral severa (ERO $0,93 \mathrm{~cm}^{2}$, VR $108 \mathrm{ml}$ ), en relación con prolapso de velo anterior (A1) y velo posterior (P1-P2). Se comprobó una imagen compatible con rotura de cuerdas, al menos de P1, y una llamativa dilatación de aorta ascendente de $46 \mathrm{~mm}$ a nivel de senos de Valsalva, Z-Score 6,51 y de 33 mm en la unión sinotubular (UST), además de la válvula aórtica trivalva con insuficiencia aórtica ligera (Figura 3 y Figura 4).

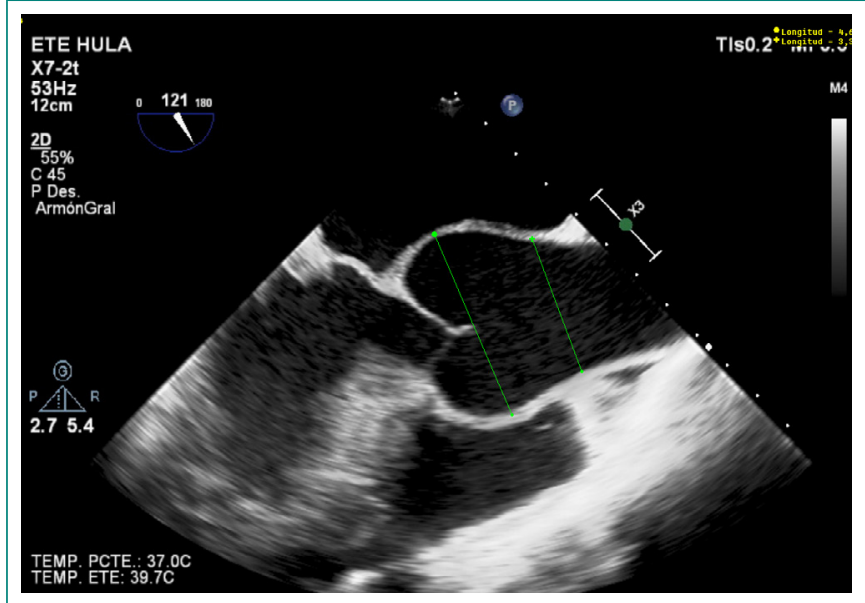

Figura 3. Imagen de ETE que muestra dilatación de raíz aórtica y UST

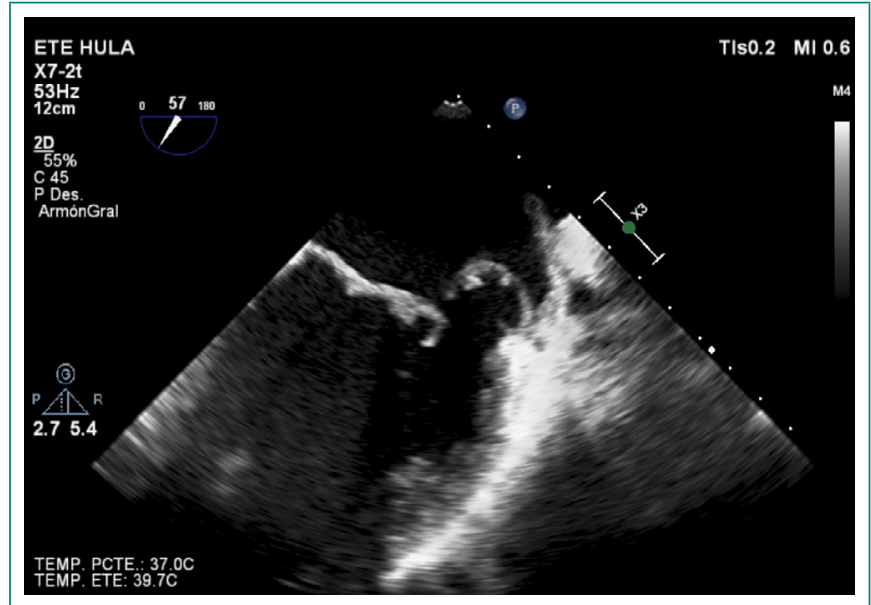

Figura 4. Imagen de ETE que muestra engrosamiento de válvula mitral y prolapso del velo posterior

\section{Diagnóstico}

El diagnóstico final fue síndrome de Marfan por la dilatación de la raíz aórtica y sus características fenotípicas.

La puntuación sistémica de Ghent de nuestra paciente tuvo un total de 12 puntos: apariencia facial característica, 1 punto; pectus excavatum, 1 punto; aracnodactilia, signos de muñeca y pulgar, 3 puntos; pie plano, 1 punto; protrusión acetabular, 2 puntos; prolapso mitral, 1 punto; estrías en abdomen, mamas y espalda, 1 punto; deformidad del retropié, 2 puntos. 
El diagnóstico se confirmó, tras el consentimiento informado de la paciente con un estudio genético posterior mediante la combinación de dos métodos: secuenciación de nueva generación/masiva (next generation sequencing [NGS]) y secuenciación por el método de Sanger. Se identificó una variante previamente no descrita en el gen FBN1: c.1120_1121delCC (p.Pro374*). Se trata de una variante que da lugar a la introducción de un codón de parada prematuro en el aminoácido Pro374, localizado en el segundo dominio TB (dominios de factor de crecimiento $\beta$ transformante que contiene en su estructura ocho cisteínas). Según la clasificación del American College of Medical Genetics $(\mathrm{ACMG})^{3}$, esta variante se podría clasificar como patogénica (PM:2 PVS:1, PM:4, PM:1.PP:4).

Finalmente, se le realizó un recambio valvular mitral por prótesis mecánica y cirugía de Bentall con tubo valvulado. Tuvo dos complicaciones posquirúrgicas: una disfunción ventricular izquierda ligera y una disfunción severa del ventrículo derecho que se achacó a una mala preservación miocárdica durante la cirugía y a la corrección de la insuficiencia mitral. Aunque en el síndrome de Marfan las guías recomiendan una intervención quirúrgica aórtica en caso de dilataciones mayores de $50 \mathrm{~mm}$, en este caso se optó por una cirugía de Bentall por presentar asociada valvulopatía mitral severa que precisaba cirugía cardíaca para recambio valvular protésico.

En la actualidad, la paciente se encuentra en seguimiento en Consulta de Insuficiencia Cardíaca, manteniéndose estable clínicamente.

\section{Discusión y conclusión}

En la literatura está bien descrito el SM, pero la presentación en el caso que hemos expuesto es infrecuente: tenía síndrome aórtico agudo, y no prolapso o rotura de cuerdas de la válvula mitral (clínica de debut más habitual). En este caso, las características fenotípicas de la paciente y la dilatación de la raíz aórtica fueron clave para su diagnóstico.

Hasta hace poco se empleaban los criterios de Ghent para el diagnóstico clínico. Se podía plantear este síndrome genético si se tenían dos sistemas afectados:

1. Dilatación de raíz aórtica $(Z \geq 2)$ y ectopia lentis.

2. Dilatación de raíz aórtica $(Z \geq 2)$ y mutación en FBN1.

3. Dilación de raíz aórtica $(Z \geq 2)$ y puntuación sistémica $\geq 7$ puntos.

4. Ectopia lentis y mutación en FBN1 asociada previamente a afectación aórtica.
En nuestro caso, la variante identificada del gen FBN1 era patogénica. Aunque no se han descrito previamente, las variantes de tipo "truncamiento" (tipo frameshift o nonsense) se consideran causantes de la enfermedad y, generalmente en esta región del gen, asociadas al desarrollo del SM.

Respecto a la correlación genotipo-fenotipo, y revisando la literatura disponible, Faivre et al. ${ }^{4}$ evaluaron una cohorte de 1.013 probandos con esta afección: determinaron que el $40 \%$ de los pacientes con variantes de "truncamiento" (frameshift o nonsense) presentaba mayor afectación esquelética, mientras que sólo el $28 \%$ con otro tipo de variantes sufría esta afectación. En este grupo, la presencia de trastornos oculares (ectopia lentis) fue menos frecuente que en otro tipo de mutaciones. La probabilidad de desarrollar dilatación aórtica en portadores de cualquier variante patogénica en FBN1 aumentaba significativamente a partir de los 20 años y era casi del 100\% a lo largo de toda la vida. Otro dato interesante de este estudio epidemiológico ${ }^{4}$ es que la edad de presentación de las manifestaciones fenotípicas, en general, se solía retrasar en las variantes de tipo "truncamiento", como ocurre en el caso de nuestra paciente, que no había tenido contacto hospitalario previo. Por tanto, sería recomendable incluir esta variante en el cribado genético familiar, ya que la identificación en familiares tiene un valor clínico predictivo de enfermedad.

Estudios epidemiológicos sugieren que los portadores de estas variantes pueden no cumplir criterios diagnósticos de la enfermedad en edades tempranas, y es conveniente realizar un seguimiento hasta edades avanzadas.

\section{Bibliografía}

1. Ganesh R, Vijayakumar R, Selvakumar H. Marfan syndrome: a case report. Case Reports in Dentistry. 2012. doi: 10.1155/2012/595343.

2. Sánchez Martínez R. Enfermedad de Marfan: revisión clinicoterapéutica y guías de seguimiento. Semin Fund Esp Reumatol. 2011; 12(4): 112-122. doi:10.1016/j.semreu.2011.09.001.

3. Richards S, Aziz N, Bale S, Bick D, Das S, Gastier-Foster J, et al. Standards and guidelines for the interpretation of sequence variants: a joint consensus recommendation of the American College of Medical Genetics and Genomics and the Association for Molecular Pathology. Genet Med. 2015 May; 17(5): 405-424. doi: 10.1038/gim.2015.30.

4. Faivre L, Collod-Beroud G, Loeys BL, Child A, Binquet C, Gautier E, et al. Effect of mutation type and location on clinical outcome in 1,013 probands with Marfan syndrome or related phenotypes and FBN1 mutations: an international study. Am J Hum Genet. 2007 Sep; 81(3): 454-466. doi: 10.1086/520125. 